\title{
REAC Neuromodulation Treatments in Depression, Anxiety and Stress. A Comparative Retrospective Study
}

This article was published in the following Dove Press journal: Psychology Research and Behavior Management

\begin{abstract}
Ana Rita Pinheiro
Barcessat $\mathbb{D}^{1}{ }^{1, *}$

Marina Nolli Bittencourt $\mathbb{D}^{2, *}$

Rebeca Góes Gonçalves (1)

Ana Vitoria Goncalves de

Oliveira Cruz $\mathbb{D}^{\prime}$

José Alfredo Coelho Pereira ${ }^{3}$

Fabio Augusto Bechelli ${ }^{4}$

Arianna Rinaldi $\mathbb{D}^{3,5}$

'Health and Biological Sciences Department, Federal University of Amapá, Macapá, Brazil; ${ }^{2}$ Department of Nursing, Federal University of Mato Grosso, Cuiabá, Brazil; ${ }^{3}$ Research Department, Rinaldi Fontani Foundation, Florence, Italy; ${ }^{4}$ International Scientific Society of Neuro Psycho Physical Optimization with REAC Technology, Brazilian Branch, São Paulo, Brazil; ${ }^{5}$ Department of Neuro Psycho Physio Pathology and Neuro Psycho Physical Optimization, Rinaldi Fontani Institute, Florence, Italy
\end{abstract}

*These authors contributed equally to this work
Correspondence: Arianna Rinaldi Department of Neuro Psycho Physio Pathology and Neuro Psycho Physical Optimization, Rinaldi Fontani Institute, Florence, Italy

Email ari@irf.it
Introduction: The purpose of this retrospective study was to compare the effects of two different modalities of administration of the neuro psycho physical optimization (NPPO) neuromodulation treatment, applied with radio electric asymmetric conveyer (REAC) biotechnology devices. Both the modalities are aimed at improving the strategies to deal with and optimize the allostatic response to environmental stressors and exposome. This allows to reduce the dysfunctional adaptive behavior patterns, which underlie many neuropsychological symptoms and pathologies, and to improve the symptoms of depression, anxiety and stress.

Materials and Methods: From a population of subjects experiencing at least two of the three symptoms depression, anxiety and stress, the selection of pre and post-treatment Depression, Anxiety, Stress 21 items scale (DASS 21) data was made proceeding with a reverse chronological recruitment mechanism, until reaching 150 subjects for each of the 2 groups. The first group was treated with the neuro psycho physical optimization treatment (NPPO), which is the punctiform modality of administration on the auricle pavilion, and the second group was treated with the neuro psycho physical optimization treatment, which is the area modality of administration applied by the planar probe on the cervicobrachial area (NPPO-CB).

Results: The Wilcoxon signs test confirmed the differences in scores in pre and posttreatment DASS-21. The comparison between the two groups data and the comparison across groups data showed that NPPO and NPPO-CB have the same efficacy in reducing the symptoms of depression, anxiety and stress, after a single treatment cycle. Statistical significance was set at $\mathrm{p}<0.05$.

Discussion: This is the first efficacy descriptive comparison between the two different modalities of administration of the NPPO treatment, as different options for the same clinical indication.

Keywords: neuromodulation, noninvasive neurostimulation, behavioral disorders, DASS-21

\section{Introduction}

Since ancient times, electricity has been used as a therapeutic technique in some mood and neurological disorders. ${ }^{1}$ Of course, at the time the nature of the electrical phenomenon was not known, nor how to artificially generate it. Despite these limitations, it had been understood that electricity produced naturally by some animals, in particular by the so-called electric fish, like torpedo rays, common in the Mediterranean Sea, could be useful to treat some health problems such as headaches, melancholy and epileptic seizures. ${ }^{1}$ Over the years, knowledge has advanced 
to create a wide availability of neuro stimulation technologies and techniques that use electric current and electromagnetic fields in various ways. Among these, the oldest is electro convulsion therapy. ${ }^{2}$ More recent techniques are trans cranial magnetic stimulation (TMS), ${ }^{3}$ direct $(\mathrm{tDCS})^{4,5}$ or alternating current stimulation (tACS), ${ }^{5,6}$ transcranial random noise stimulation (tRNS), ${ }^{5,7}$ transcranial focused ultrasound ${ }^{8}$ and stimulation with radio electric asymmetrically conveyed fields (REAC). ${ }^{9,10}$ Each of these techniques in the context of the same therapeutic indications has various methods of therapeutic administration. ${ }^{11-13}$

In the context of REAC technology, the administration of neuromodulation treatments is carried out with electrodes called Asymmetric Conveyer Probe (ACP). These can be of punctiform type to treat small areas, or planar type for larger areas. REAC neuromodulation treatments for depression, anxiety and stress symptoms ${ }^{14-21}$ are identified with the generic definition of Neuro Psycho Physical Optimization (NPPO). NPPO is the term that is commonly used for the treatment with punctiform ACP administered on the auricle, while that administered with planar ACP in the cervical region is called NPPO-CB, as described in a previous work carried out as an arm of the same research project. $^{22}$

The NPPO treatment takes about three seconds, being designed to be used on large populations, ie, in social prevention projects against behaviors and symptoms related to depression, anxiety and stress. ${ }^{9}$ The NPPO-CB requires about 4 minutes and is preferably used when the symptoms related to a depressive, anxious or stress state, occur with tension in the shoulders and neck. In fact, the correlation between depression, anxiety, stress, and the cervical/cervicobrachial area is well described in the literature. $^{23-30}$ Furthermore, it has been shown that this correlation is present in all ages. ${ }^{25,27}$

The purpose of this study was analyzing retrospectively the data obtained from the psychometric test depression Anxiety Stress Scale 21 items (DASS-21) ${ }^{31}$ in the NPPO and NPPO-CB groups. The psychometric test results were compared before and after the treatment administration in each group. The final analysis aimed to compare the difference in effectiveness between NPPO and NPPO-CB groups.

\section{Materials and Methods}

\section{Study Design}

A retrospective study is defined as a study in which the research question was determined after the data were collected. This is a retrospective, non-randomized, nonblinded study, aimed to compare retrospectively the efficacy of 2 administration modalities of neuro psycho physical optimization treatment (NPPO and NPPO-CB) in depression, anxiety and stress, evaluating the results through the use of DASS-21 test. DASS-21 is a validated test, which evaluates the severity of mental disorder symptoms associated to depression, anxiety, and stress, using a severity scale that ranges from normal to mild, moderate, severe and extremely severe. In order to reach high levels of evidence, the design of the study was based on this specific inclusion criterion: to be positive to at least two clusters out of three of DASS-21 test, that means that the test results of the recruited subjects had to exceed the threshold of normality in 2 out of 3 clusters. Consequently, on a population of about 300 subjects for each administration modality group, the subjects were recruited in reverse chronological order, starting from the most recent treated, excluding those who matched exclusion criteria (age, pregnancy, diagnosis of psychiatric diseases, ongoing psychopharmacological or psychotherapeutic treatments), up to reach the number of 150 subjects for each group, because this cohort size was assumed to be sufficient for the study design.

\section{Context of the Study and Data Acquisition}

The context for data acquisition is the REACNeuromodulation research project. The aim of this project is to evaluate the effects of neuromodulation treatments with REAC technology in the epigenetic and neuropsychological fields on anxiety, depression and stress, in the Federal University of Amapá (UNIFAP) community.

\section{Ethics}

In the ambit of the research project REAC Neuromodulation, this study was approved by the ethical committee of the Federal University of Amapá with opinion number 3.640.674 dated October 14, 2019. The study was conducted in full compliance with the Declaration of Helsinki - Ethical principles for medical research involving human subjects. Informed consent was obtained from all participants.

\section{Subjects}

The inclusion criteria used for the selection of subjects to be included in the retrospective analysis were as follows: 
both genders, age between 18 and 70 years, positivity to at least two out the three clusters depression, anxiety and stress of the DASS-21 test. The exclusion criteria were: pregnancy, diagnosis of psychiatric diseases, ongoing psychopharmacological or psycho-therapeutic treatments. Proceeding with a reverse chronological recruitment mechanism, the selection was continued until reaching 150 subjects for each of the 2 groups, the first treated with the NPPO treatment and the second treated with the NPPO-CB treatment.

The prescription of NPPO-CB administration modality instead of NPPO was preferred when, through clinical examination, the doctor observed cervical brachial contraction and the forward head-neck posture.

The NPPO group consisted of 150 people, 57 men $(38 \%)$, with an average age of $51.1 \pm 12.65$ years, and 93 women $(62 \%)$, with an average age of $49.8 \pm 13.81$ years. The overall average age of the group was $50.3 \pm$ 13.35 years. The NPPO-CB group consisted of 150 people, 66 men (44\%), with an average age of $48.7 \pm 14.25$ years, and 84 women (56\%), with an average age of $45.8 \pm$ 16.14 years. The overall average age of the group was 47.1 \pm 15.35 years.

After the DASS-21 test (T0), all the subjects underwent a preliminary REAC neuromodulation treatment called Neuro Postural Optimization (NPO) ${ }^{10,32-36}$ (T1). After at least an hour after the NPO treatment, the subjects started the NPPO or NPPO-CB 18 sessions treatment cycle (T2). The time interval between one session and the next was at least one hour, up to a maximum of 4 sessions per day. The entire treatment cycle of 18 sessions was completed within 4 weeks. At the last of the 18 sessions of NPPO or NPPO-CB treatment cycle, the subjects repeated the DASS 21 test (T3). The time points for the comparison of the DASS-21 test were T0 and T3.

\section{DASS 21 Test}

The DASS-21 is a 21-item self-report instrument designed by Syd Lovibond and Peter Lovibond in 1995. It was originally developed to provide maximum differentiation between depressive and anxious symptoms. ${ }^{31}$ This psychometric test is a measure of mental health, focused on the three traits of depression, anxiety and stress. Compared to the DSM-IV classification, the DASS Anxiety scale matches closely the criteria for various Anxiety Disorders, with the exception of Generalized Anxiety Disorder (GAD). The DASS Depression scale, even if it is not specific to depression, matches closely the criteria of the Mood Disorders. The DASS Stress scale matches quite closely the DSM-IV symptom criteria for GAD. The test consists of a list of 21 items, each of which is to be rated on a four-point scale. It assesses difficult relaxing, nervous arousal, and being easily upset/agitated, irritable/overreactive and impatient.

\section{REAC Technology}

REAC Technology is based on specific environmental radioelectric fields emitted by the device, which interact with the bioelectric field of the subject being treated inducing microcurrents in his body. The specific probe ACP, which connects the device to the body of the patient, allows the radio electric field emitted by the REAC device to interact with all the biological structures that contain electrical charges, such as the cells and human body. This interaction induces endogenous microcurrents in them. This scheme has been developed to create an asymmetric circuit, that underlies the cell polarity, ${ }^{37}$ and neuro transmission. $^{38,39}$ The REAC neuromodulation treatments are based on a pulsed $5.8 \mathrm{GHz}$ radio electric emission. Each treatment protocol is pre-programmed by the manufacturer and cannot be modified by the operator. The REAC technology devices used in this retrospective analysis were BENE 110 universal model (ASMED, Florence, Italy).

\section{REAC Neuromodulation Treatments REAC Neuro Postural Optimization Treatment}

The REAC-NPO neuromodulation treatment has shown to be able to optimize the neurotransmission in the whole encephalic mass, determining a more functional and efficient response, ${ }^{10,32,34}$ even in neurodegenerative diseases such as Parkinson's and Alzheimer's. ${ }^{34-36}$ The REACNPO consists of a single treatment administered on a specific area of the auricle scapha.

\section{REAC Neuro Psycho Physical Optimization Treatments}

NPPO treatment has been designed to optimize the individual response to environmental stressors, that can induce a wide range of disorders and pathologies, as depression, anxiety, and stress.

The increased muscle tension, detectable in the cervico brachial region, ${ }^{24,40}$ and originating in this alarm/stress reaction, is the physiological rationale that explains why in the NPPO-CB treatment, the ACP is applied on the cervicobrachial area. 


\section{Statistical Analysis}

The data of the total 300 patients, grouped by type of treatment into two groups of 150 , were sent to the analysis of a statistician, who was blind to the treatment administered.

For statistical analysis was used IBM Statistical Package for Social Science (SPSS) 22. Summary statistics of number of patients and percentages in the five categories (normal, mild, moderate, severe, extremely severe) at the two time points $\mathrm{T} 0$ and $\mathrm{T} 3$ for the three clusters (depression, anxiety, stress) were performed. The Wilcoxon Signs test was performed to confirm the differences in scores between $\mathrm{T} 0$ and $\mathrm{T} 3$, as reported in Table 1. Statistical significance was set at $\mathrm{p}<0.05$. For both the tests and in both the groups statistical significance was found at $\mathrm{p}<0.005$.

\section{Results NPPO Group}

At T0 DASS-21 test, the NPPO group reported the following results: for the cluster depression, 25 patients $(16,7 \%)$ in normal category, $31(20,7 \%)$ in mild, $43(28,7 \%)$ in moderate, $26(17,3 \%)$ in severe, $25(16,7 \%)$ in extremely severe; for the cluster anxiety, 21 (14\%) patients at normal level, $18(12 \%)$ at mild level, $39(26 \%)$ at moderate, 21 $(14 \%)$ at severe, $51(34 \%)$ at extremely severe; as regards the cluster stress, $12(8 \%)$ patients were at normal level, 24 $(16 \%)$ at mild level, $42(28 \%)$ at moderate, $40(26,7 \%)$ at severe, $32(21,3 \%)$ at extremely severe.

At T3, about 4 weeks from the start of treatment, NPPO group reported the following results: for the cluster depression, $94(62,7 \%)$ patients in normal category, $23(15,3 \%)$ in mild, $22(14,7 \%)$ in moderate, $8(5,3 \%)$ in severe, $3(2 \%)$ in extremely severe; for the cluster anxiety, $85(56,7 \%)$ patients at normal level, $12(8 \%)$ at mild level, $28(18,7 \%)$ at moderate, $13(8,7 \%)$ at severe, $12(8 \%)$ at extremely severe; as regards the cluster stress, $84(56 \%)$ patients were at normal level, $28(18,7 \%)$ at mild level, $22(14,7 \%)$ at moderate, 12 $(8 \%)$ at severe, $4(2.7 \%)$ at extremely severe.

Figure 1 shows how the neuro psycho physical optimization treatment in NPPO administration modality is effective in reducing the symptoms of depression, anxiety and stress, comparing the results of the DASS-21 tests carried out before and after the treatment for each of the 3 clusters.

Table I Wilcoxon Signed-Rank Test Results

\begin{tabular}{|c|c|c|c|}
\hline \multicolumn{4}{|c|}{ NPPO Modality of Treatment Administration $(N=150)$} \\
\hline \multicolumn{4}{|l|}{ SIGN TEST } \\
\hline & Depression post - Depression pre & Anxiety post - Anxiety pre & Stress post - Stress pre \\
\hline Z & -10.417 & -10.083 & -11.161 \\
\hline Asymp. Sig. (2-tailed) & 0.000 & 0.000 & 0.000 \\
\hline \multicolumn{4}{|l|}{ WILCOXON } \\
\hline & Depression post - Depression pre & Anxiety post - Anxiety pre & Stress post - Stress pre \\
\hline Z & $-10.02 \mid$ & -9.482 & -10.133 \\
\hline Asymp. Sig. (2-tailed) & 0.000 & 0.000 & 0.000 \\
\hline \multicolumn{4}{|c|}{ NPPO-CB MODALITY OF TREATMENT ADMINISTRATION (N = I50) } \\
\hline \multicolumn{4}{|l|}{ SIGN TEST } \\
\hline & Depression post - Depression pre & Anxiety post - Anxiety pre & Stress post - Stress pre \\
\hline Z & -10.780 & -10.676 & -11.007 \\
\hline Asymp. Sig. (2-tailed) & 0.000 & 0.000 & 0.000 \\
\hline \multicolumn{4}{|l|}{ WILCOXON } \\
\hline & Depression post - Depression pre & Anxiety post - Anxiety pre & Stress post - Stress pre \\
\hline Z & -9.848 & -9.873 & -10.097 \\
\hline Asymp. Sig. (2-tailed) & 0.000 & 0.000 & 0.000 \\
\hline
\end{tabular}

Abbreviations: DASS 2I, Depression Anxiety Stress Scale; NPO, neuro postural optimization; NPPO, neuro psycho physical optimization; NPPO-CB, neuro psycho physical optimization-cervico brachial; ACP, asymmetric conveyer probe. 

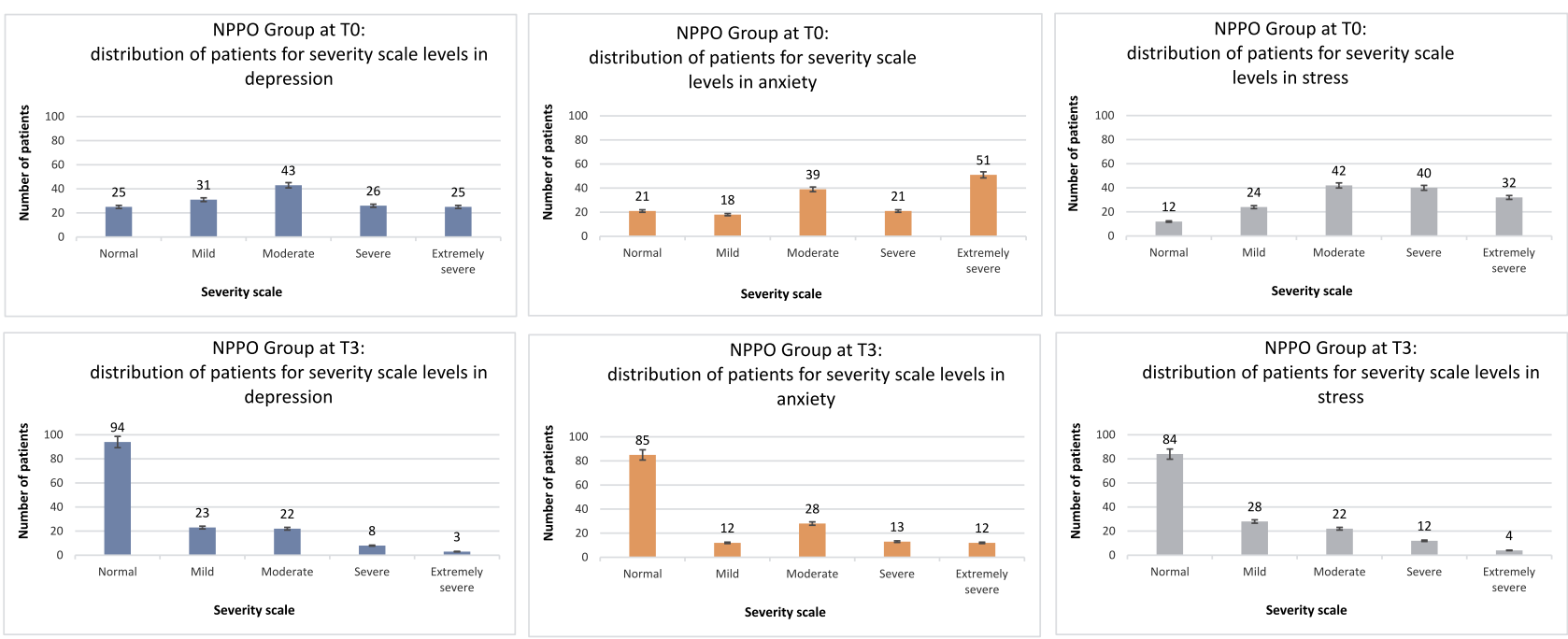

Figure I NPPO modality of treatment administration: efficacy in the reduction of the symptoms in the three clusters, from T0 to T3.

\section{NPPO-CB Group}

At T0 test, the NPPO-CB group reported the following results: for the cluster depression, $32(21,3 \%)$ patients in normal category, $26(17,3 \%)$ in mild, $48(32 \%)$ in moderate, $19(12,7 \%)$ in severe, $25(16,7 \%)$ in extremely severe; for the cluster anxiety, $18(12 \%)$ patients at normal level, $24(16 \%)$ at mild level, $43(28,7 \%)$ at moderate, 23 $(15,3 \%)$ at severe, $42(28 \%)$ at extremely severe; as regards the cluster stress, $17(11,3 \%)$ patients were at normal level, $31(20,7 \%)$ at mild level, $39(26 \%)$ at moderate, $36(24 \%)$ at severe, $27(18 \%)$ at extremely severe.

At T3, about 4 weeks from the start of treatment, NPPO-CB group reported the following results: for the cluster depression, $95(63,3 \%)$ patients in normal category,
$26(17,3 \%)$ in mild, $19(12,7 \%)$ in moderate, $6(4 \%)$ in severe, $4(2,7 \%)$ in extremely severe; for the cluster anxiety, $89(59,3 \%)$ patients at normal level, $16(10,7 \%)$ at mild level, $32(21,3 \%)$ at moderate, $10(6,7 \%)$ at severe, 3 $(2 \%)$ at extremely severe; as regards the cluster stress, 100 $(66,7 \%)$ patients were at normal level, $21(14 \%)$ at mild level, $20(13,3 \%)$ at moderate, $6(4 \%)$ at severe, $3(2 \%)$ at extremely severe.

Figure 2 shows the efficacy of the neuro psycho physical optimization treatment in NPPO-CB modality of administration in reducing the symptoms of depression, anxiety and stress, comparing the results of the DASS- 21 tests carried out before and after the treatment for each of the 3 clusters.

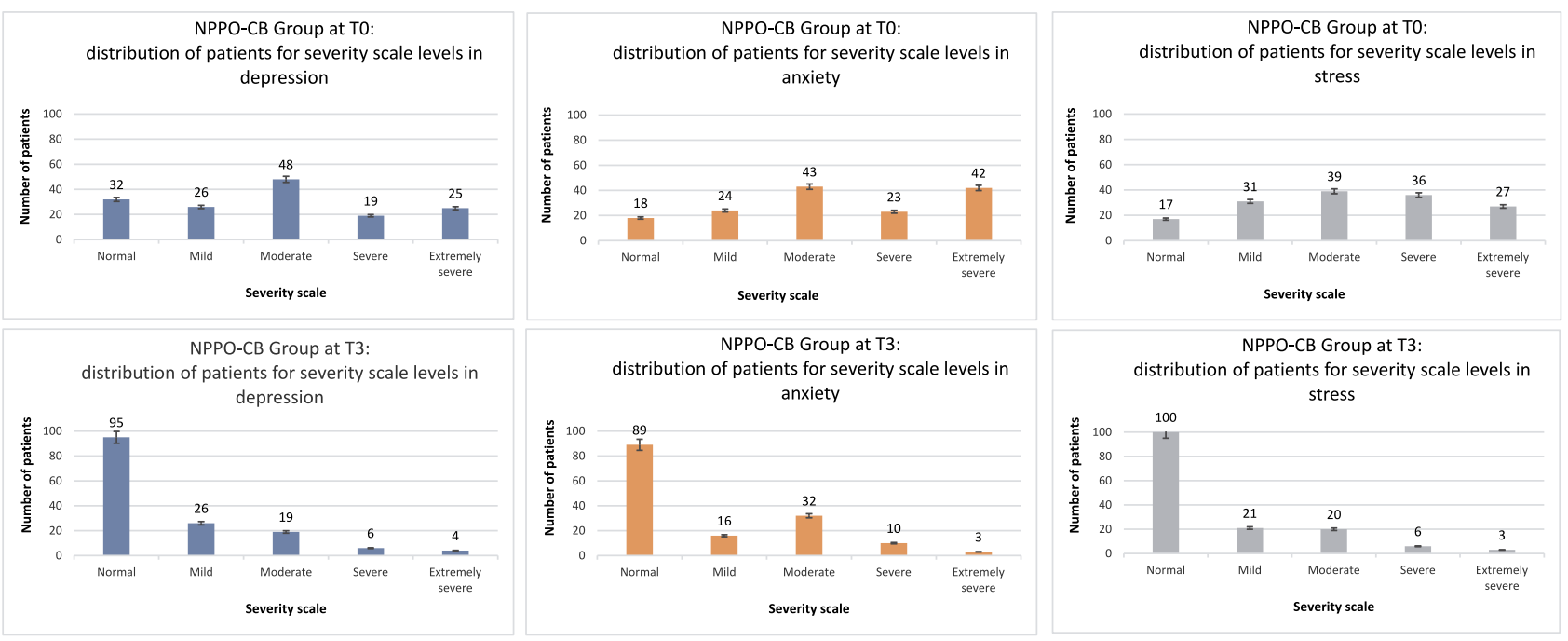

Figure 2 NPPO-CB modality of treatment administration: efficacy in the reduction of the symptoms in the three clusters, from T0 to T3. 


\section{Comparison Between the Two Modalities of Treatment Administration}

The results obtained in the 2 groups treated with the 2 modalities of treatment administration can be superimposed in all 3 clusters subject to evaluation of the DASS-21 test.

In fact, as regards the depression cluster, in the NPPO group $83.3 \%$ (125/150) of the patients had the symptom at T0, while after the treatment the percentage dropped to $37.3 \%(56 / 150)$. Similar results for patients in the NPPOCB group, with $78.6 \%$ (118/150) positive for the symptom depression at $\mathrm{T} 0$ and $36.6 \%(55 / 150)$ at $\mathrm{T} 3$.

As regards the anxiety cluster, in the NPPO group, $86 \%(129 / 150)$ of the patients had the symptom at $\mathrm{T} 0$, while after the treatment the percentage dropped to $43.3 \%$
(65/150). Similar results for patients in the NPPO-CB group, with $88 \%(132 / 150)$ positive for the symptom anxiety at T0 and $40.6 \%(61 / 150)$ at $\mathrm{T} 3$.

Finally, as regards the stress cluster, 92\% (138/150) of patients of NPPO group were positive to the symptom at $\mathrm{T} 0$, while the percentage dropped to $37.3 \%(56 / 150)$ at $\mathrm{T} 3$. In the NPPO-CB group, $88.6 \%(133 / 150)$ were positive at $\mathrm{T} 0$ and $33.3 \%(50 / 150)$ at $\mathrm{T} 3$.

These results show the similar efficacy of the 2 modalities of administration of the NPPO treatment. In fact, Figures 3 and 4 highlight how at T3 the median tends to move towards lower symptom severity scores compared to T0, in all 3 clusters and in both the modalities of administration. Moreover, the dispersion from the median tends

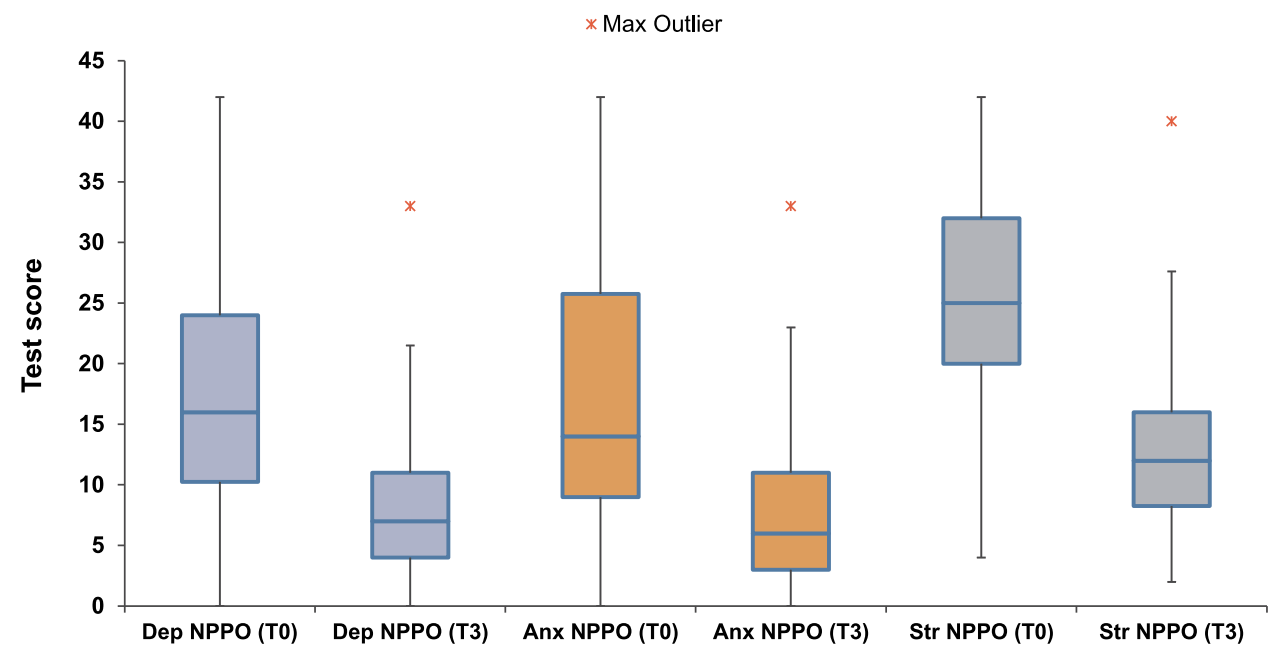

Figure 3 NPPO modality of treatment administration: comparison between T0 and T3 in the three clusters.

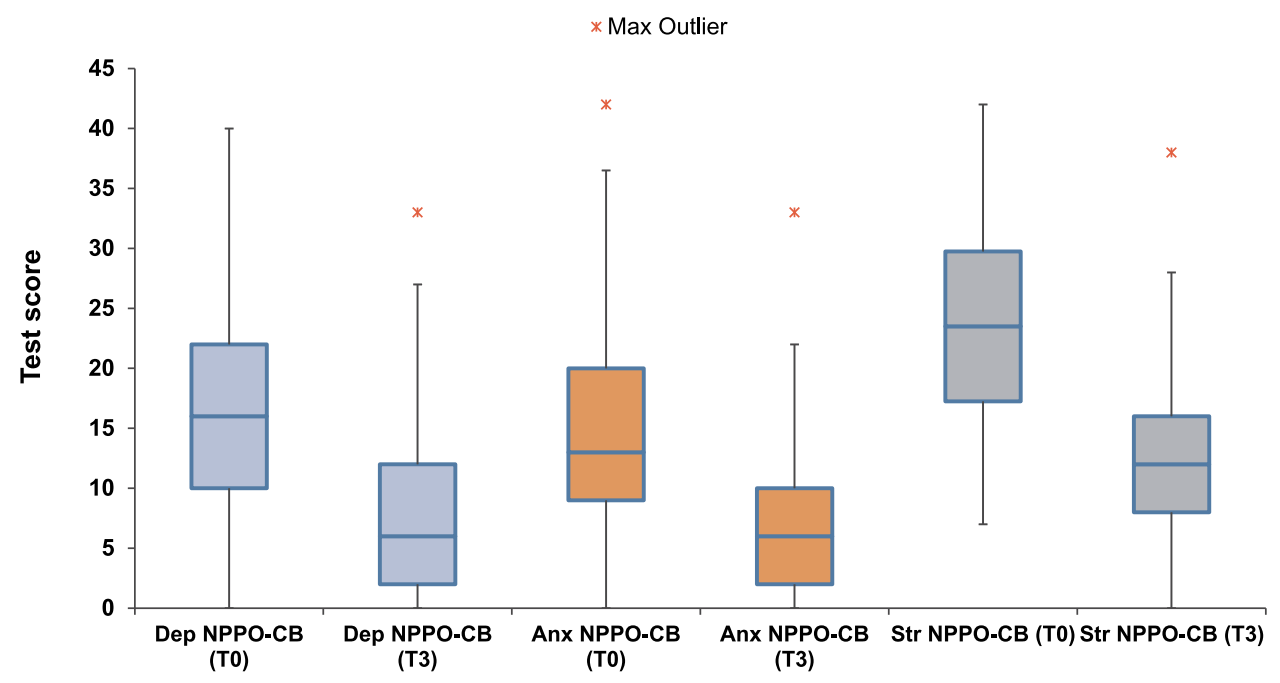

Figure 4 NPPO-CB modality of treatment administration: comparison between T0 and T3 in the three clusters. 


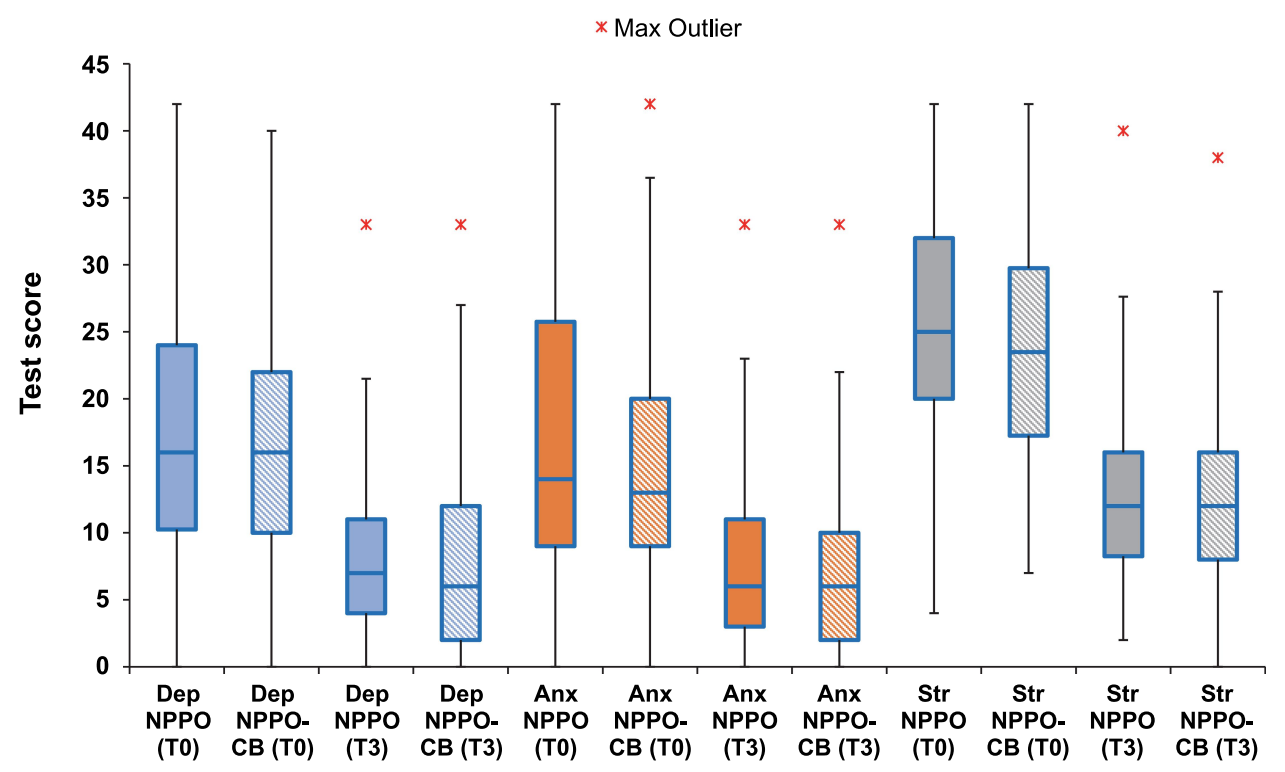

Figure 5 Effectiveness comparison across groups. Results at T0 and T3 compared across NPPO and NPPO-CB treatment modalities in each cluster.

to decrease, at $\mathrm{T} 3$ compared to $\mathrm{T} 0$, highlighting the concentration trend of the subjects around lower severity levels. Figure 5 shows the effectiveness comparison across groups. Results at T0 and T3 are compared across NPPO and NPPO-CB treatment modalities in each cluster.

\section{Discussion}

The feeling of tension and pain in the neck and shoulders in relation to a depressive, anxious or stressful episode is a common experience in the life of any individual. To treat this symptomatic picture, various approaches have been proposed, such as cervical treatments with transcutaneous electrical nerve stimulation ${ }^{41}$ (TENS), hot pack $^{41}$ (HP), pulsed electro magnetic fields ${ }^{41}$ (PEMF), far-infrared radiation $^{42}$ (FIR), low-frequency stimulation ${ }^{42,43}$ (LFS), acupuncture, ${ }^{42,43}$ and physiotherapy. ${ }^{44,45}$

Unexpectedly, these treatments ${ }^{41-45}$ aimed at treating a predominantly physical symptom, such as the sensation of tension and pain in the neck and shoulders, determined also a psycho-emotional effect, resulting in an improvement in the depressive, anxious and stressful state.

The physiological rationale behind this symptomatic correlation could be identified in the alarm reaction, ${ }^{46-48}$ as an initial response that the body experiences when under stress. This response is known as startle response ${ }^{49,50}$ when triggered by acoustic stimuli, or avoidance response ${ }^{51}$ when it is aimed at preventing an aversive stimulus.

In humans, as in all animals, the alarm reaction is a fundamentally unconscious defensive reaction in response to unexpected, sudden, or threatening cues, characterized by increased autonomic and neuroendocrine activation, and specific behavior patterns that also involve postural attitude. ${ }^{47,48}$ The alarm/stress reaction is initially and subconsciously processed by the low road of Le Doux. ${ }^{52,53}$ In the low road, the process of elaborating the external stimuli implies that these are initially received by the sense organs, conveyed to the thalami and sent by them to the amygdales, for subsequent processing of information.

From here, new bioelectric impulses impart signals to the effector organs for the prompt reaction of the body in terms of behavior and action aimed at expressing emotion. The behavior developed by the low road has its own specific function from the point of view of survival, ie, it is better to react to potentially dangerous circumstances as if they were real, rather than not to react at all. Therefore, the low road is a way through which the signal is processed more quickly, but also less detailed. It is a path that allows us to quickly activate our body, to respond promptly to a threat. All this leads the whole organism to maintain an alarm response, not consciously perceived by the individual, but manifested with various postural attitudes, such as the forward head/neck posture. ${ }^{54}$

Subsequently, the alarm/stress reaction is always unconsciously processed by the high road of Le Doux. ${ }^{52,53}$ The process of elaborating the external stimuli in the high road implies that these are initially received by the sense organs, conveyed to the thalami and sent by them to the neocortex. From the cerebral neocortex, new 
impulses are generated and sent to the amygdales, which process and restart new impulses towards the effector organs for emotional response and other body reactions. Hence, the elaboration of the environmental stimuli through the high road, allows the higher cognitive systems of the brain to carry out a more detailed evaluation of the stimulus, including relationships with other stimuli and representations of past experiences, which lead to a more modulated emotional response. We can summarize the concept of the high road as a way that uses the cortical functions in response to external stimuli.

The REAC NPPO and NPPO-CB treatment modalities have been developed almost simultaneously. The NPPO treatment was basically designed to optimize the alarm response mediated by the high road of Le Doux, ${ }^{52,53}$ while the NPPO-CB was designed for the response elaborated by the low road.

On the basis of clinical observation and anamnesis, subjects were treated with NPPO-CB when showed and/ or reported a state of cervicobrachial tension, sometimes with evident postural alterations of the neck. These symptoms were evaluated as a low road mediated alarm response. $^{52,53}$

The NPPO modality has been the most used in clinical practice, probably due to the rapidity of the administration time, which is overall some seconds, compared to about four minutes of the NPPO-CB modality. Another aspect that made NPPO-CB treatment modality less used was the difficulty of preparing and positioning the previous aluminum ACP on the cervicobrachial region. Thanks to the technological innovation, that has allowed the production of new ACPs with particularly soft materials and predefined forms, the NPPO-CB administration modality is faster for the doctor and more comfortable for the patient. This innovation has allowed a greater diffusion of NPPO$\mathrm{CB}$ in the clinical use.

This study was conducted with a convenience sampling, as subjects were included exclusively in reverse chronological order, starting from the most recent treated. To reduce the biases dependent on this type of sampling, those who analyzed the data were blinded to the method of administration of the treatment. The sample size was not calculated on formal hypotheses considering the descriptive nature of the study and the number of patients in each group was estimated based on the available data. This is the first efficacy descriptive comparison between NPPO and NPPO-CB modalities, as different option of administration for the same clinical indication.
Analyzing the DASS-21 test results, we can affirm that the results of the two groups are substantially superimposable and that NPPO and NPPO-CB have the same effectiveness in reducing depression, anxiety and stress, after a single treatment cycle.

The limitation of this study is inherent in its nature as a retrospective study, as these studies are based solely on the use of data collected in the past from pre-existing groups of subjects. All this does not allow to have sham control. To overcome these limitations, these studies usually try to investigate the largest possible sample, as we did in our study.

\section{Author Contributions}

All authors made a significant contribution to the work reported, whether that is in the conception, study design, execution, acquisition of data, analysis and interpretation, or in all these areas; took part in drafting, revising or critically reviewing the article; gave final approval of the version to be published; have agreed on the journal to which the article has been submitted; and agree to be accountable for all aspects of the work.

\section{Funding}

This research did not receive any specific grant from funding agencies in the public, commercial, or not-forprofit sectors.

\section{Disclosure}

A.R. is the daughter of Salvatore Rinaldi and Vania Fontani, authors of the REAC technology patent and reports no other potential conflicts of interest for this work. The other authors declare no conflicts of interest.

\section{References}

1. Tsoucalas G, Karamanou M, Lymperi M, Gennimata V, Androutsos G. The "torpedo" effect in medicine. Int Marit Health. 2014;65(2):65-67. doi:10.5603/IMH.2014.0015

2. Gazdag G, Ungvari GS. Electroconvulsive therapy: 80 years old and still going strong. World J Psychiatry. 2019;9(1):1-6. doi:10.5498/wjp. v9.i1.1

3. Perera T, George MS, Grammer G, Janicak PG, Pascual-Leone A, Wirecki TS. The clinical TMS society consensus review and treatment recommendations for TMS therapy for major depressive disorder. Brain Stimul. 2016;9(3):336-346. doi:10.1016/j.brs.2016.03.010

4. Lefaucheur JP, Antal A, Ayache SS, et al. Evidence-based guidelines on the therapeutic use of transcranial direct current stimulation (tDCS). Clin Neurophysiol. 2017;128(1):56-92. doi:10.1016/j.clinph.2016.10. 087

5. Moreno-Duarte I, Gebodh N, Schestatsky P, et al. Transcranial electrical stimulation. In: Cohen Kadosh R, editor. The Stimulated Brain. San Diego: Academic Press; 2014:35-59. 
6. Antal A, Paulus W. Transcranial alternating current stimulation (tACS). Front Hum Neurosci. 2013;7:317. doi:10.3389/fnhum.2013. 00317

7. Moret B, Donato R, Nucci M, Cona G, Campana G. Transcranial random noise stimulation (tRNS): a wide range of frequencies is needed for increasing cortical excitability. Sci Rep. 2019;9 (1):15150. doi:10.1038/s41598-019-51553-7

8. Di Biase L, Falato E, Di Lazzaro V. Transcranial focused ultrasound (tFUS) and transcranial unfocused ultrasound (tUS) neuromodulation: from theoretical principles to stimulation practices. Front Neurol. 2019;10:549. doi:10.3389/fneur.2019.00549

9. Rinaldi A, Rinaldi C, Coelho Pereira JA, et al. Radio electric asymmetric conveyer neuromodulation in depression, anxiety, and stress. Neuropsychiatr Dis Treat. 2019;15:469-480. doi:10.2147/NDT. S195466

10. Rinaldi S, Mura M, Castagna A, Fontani V. Long-lasting changes in brain activation induced by a single REAC technology pulse in wi-fi bands. Randomized double-blind fMRI qualitative study. Sci Rep. 2014;4(1):5668. doi:10.1038/srep05668

11. McClintock SM, Reti IM, Carpenter LL, et al. Consensus recommendations for the clinical application of repetitive transcranial magnetic stimulation (rTMS) in the treatment of depression. J Clin Psychiatry. 2018;79(1):35-48. doi:10.4088/JCP.16cs10905

12. Monte-Silva K, Kuo MF, Liebetanz D, Paulus W, Nitsche MA. Shaping the optimal repetition interval for cathodal transcranial direct current stimulation (tDCS). J Neurophysiol. 2010;103(4):1735-1740. doi:10.1152/jn.00924.2009

13. Galvez V, Alonzo A, Martin D, Loo CK. Transcranial direct current stimulation treatment protocols: should stimulus intensity be constant or incremental over multiple sessions? Int J Neuropsychopharmacol. 2013;16(1):13-21. doi:10.1017/S1461145712000041

14. Rinaldi S, Fontani V, Aravagli L, Mannu P. Psychometric evaluation of a radio electric auricular treatment for stress related disorders: a double-blinded, placebo-controlled controlled pilot study. Health Qual Life Outcomes. 2010;8(1):31. doi:10.1186/1477-75258-31

15. Rinaldi S, Fontani V, Aravagli L, Margotti ML. Psychological and symptomatic stress-related disorders with radio-electric treatment: psychometric evaluation. Stress Health. 2010;26(5):350-358. doi:10.1002/smi.1298

16. Rinaldi S, Fontani V, Moretti E, et al. A new approach on stressrelated depression and anxiety: neuro-psycho- physical-optimization with radio electric asymmetric-conveyer. Indian $J$ Med Res. 2010;132:189-194.

17. Fontani V, Mannu P, Castagna A, Rinaldi S. Social anxiety disorder: radio electric asymmetric conveyor brain stimulation versus sertraline. Patient Prefer Adherence. 2011;5:581-586.

18. Fontani V, Rinaldi S, Aravagli L, Mannu P, Castagna A, Margotti ML. Noninvasive radioelectric asymmetric brain stimulation in the treatment of stress-related pain and physical problems: psychometric evaluation in a randomized, single-blind placebo-controlled, naturalistic study. Int J Gen Med. 2011;4:681-686.

19. Olivieri EB, Vecchiato C, Ignaccolo N, et al. Radioelectric brain stimulation in the treatment of generalized anxiety disorder with comorbid major depression in a psychiatric hospital: a pilot study. Neuropsychiatr Dis Treat. 2011;7:449-455.

20. Rinaldi S, Fontani V, Aravagli L, et al. Stress-related psycho-physiological disorders: randomized single blind placebo controlled naturalistic study of psychometric evaluation using a radio electric asymmetric treatment. Health Qual Life Outcomes. 2011;9(1):54. doi:10.1186/1477-7525-9-54

21. Fontani V, Aravagli L, Margotti ML, Castagna A, Mannu P, Rinaldi S. Neuropsychophysical optimization by REAC technology in the treatment of: sense of stress and confusion. Psychometric evaluation in a randomized, single blind, sham-controlled naturalistic study. Patient Prefer Adherence. 2012;6:195-199.
22. Pinheiro Barcessat ARNBM, Duarte Ferreira L, de Souza Neri E, Coelho Pereira JA, Bechelli F, Rinaldi A. REAC cervicobrachial neuromodulation treatment of depression, anxiety, and stress during the COVID-19 pandemic. Psychol Res Behav Manag. 2020;13:929937. doi:10.2147/PRBM.S275730

23. Blozik E, Laptinskaya D, Herrmann-Lingen C, et al. Depression and anxiety as major determinants of neck pain: a cross-sectional study in general practice. BMC Musculoskelet Disord. 2009;10(1):13. doi:10.1186/1471-2474-10-13

24. Elbinoune I, Amine B, Shyen S, Gueddari S, Abouqal R, HajjajHassouni N. Chronic neck pain and anxiety-depression: prevalence and associated risk factors. Pan Afr Med J. 2016;24:89. doi:10.11604/ pamj.2016.24.89.8831

25. Talvari A, Nemati N, Sini ZK, Golsefidi FN, Varkiani ME. The association of neck pain with depression and anxiety symptoms in elderly. Procedia Soc Behav Sci. 2013;82:366-368. doi:10.1016/j. sbspro.2013.06.276

26. MacDowall A, Robinson Y, Skeppholm M, Olerud C. Anxiety and depression affect pain drawings in cervical degenerative disc disease. Ups J Med Sci. 2017;122(2):99-107. doi:10.1080/03009734.2017.13 19441

27. Silva HA, Passos MP, Oliveira VA, Silva YA, Pitangui ACR, Araújo RC. Prevalence of anxiety, depression and stress symptoms and its association with neck/shoulder pain in adolescents athletes. Motricidade. 2017;13(4):13-22. doi:10.6063/motricidade.9703

28. Reichborn-Kjennerud T, Stoltenberg C, Tambs K, et al. Back-neck pain and symptoms of anxiety and depression: a population-based twin study. Psychol Med. 2002;32(6):1009-1020. doi:10.1017/ S0033291702005950

29. Veer V, Kia S, Papesch M. Anxiety and depression in head and neck out-patients. J Laryngol Otol. 2010;124(7):774-777. doi:10.1017/ S0022215110000502

30. Liu F, Fang T, Zhou F, et al. Association of depression/anxiety symptoms with neck pain: a systematic review and meta-analysis of literature in China. Pain Res Manag. 2018;2018:3259431. doi:10.1155/2018/3259431

31. Lovibond PF, Lovibond SH. The structure of negative emotional states: comparison of the depression anxiety stress scales (DASS) with the beck depression and anxiety inventories. Behav Res Ther. 1995;33(3):335-343. doi:10.1016/0005-7967(94)00075-U

32. Rinaldi S, Fontani V, Castagna A. Brain activity modification produced by a single radioelectric asymmetric brain stimulation pulse: a new tool for neuropsychiatric treatments. Preliminary fMRI study. Neuropsychiatr Dis Treat. 2011;7:649-654. doi:10.2147/NDT. S26123

33. Fontani V, Rinaldi S, Castagna A, Margotti ML. Noninvasive radioelectric asymmetric conveyor brain stimulation treatment improves balance in individuals over 65 suffering from neurological diseases: pilot study. Ther Clin Risk Manag. 2012;8:73-78.

34. Mura M, Castagna A, Fontani V, Rinaldi S. Preliminary pilot fMRI study of neuropostural optimization with a noninvasive asymmetric radioelectric brain stimulation protocol in functional dysmetria. Neuropsychiatr Dis Treat. 2012;8:149-154.

35. Olazaran J, Gonzalez B, Lopez-Alvarez J, et al. Motor effects of REAC in advanced alzheimer's disease: results from a pilot trial. $J$ Alzheimers Dis. 2013;36(2):297-302. doi:10.3233/JAD-130077

36. Olazaran J, Gonzalez B, Osa-Ruiz E, et al. Motor effects of radio electric asymmetric conveyer in alzheimer's disease: results from a cross-over trial. J Alzheimers Dis. 2014;42(1):325-332. doi:10.3233/ JAD-140417

37. Cibrian-Uhalte E, Abdelilah-Seyfried S. Cell polarity. In: Encyclopedic Reference of Genomics and Proteomics in Molecular Medicine. Berlin, Heidelberg: Springer Berlin Heidelberg; 2006:237-243.

38. Qiu C, Shivacharan RS, Zhang M, Durand DM. Can neural activity propagate by endogenous electrical field? J Neurosci. 2015;35 (48):15800-15811. doi:10.1523/JNEUROSCI.1045-15.2015 
39. Faber DS, Pereda AE. Two forms of electrical transmission between neurons. Front Mol Neurosci. 2018;11:427. doi:10.3389/fnmol.2018. 00427

40. Roman-Liu D, Grabarek I, Bartuzi P, Choromanski W. The influence of mental load on muscle tension. Ergonomics. 2013;56(7):11251133. doi:10.1080/00140139.2013.798429

41. Hattapoğlu E, Batmaz İ, Dilek B, Karakoç M, Em S, Çevik R. Efficiency of pulsed electromagnetic fields on pain, disability, anxiety, depression, and quality of life in patients with cervical disc herniation: a randomized controlled study. Turk J Med Sci. 2019;49 (4):1095-1101. doi:10.3906/sag-1901-65

42. Matsui T, Fujimoto T. Treatment for depression with chronic neck pain completely cured in $94.2 \%$ of patients following neck muscle treatment. Neurosci Med. 2011;2(2):71-77. doi:10.4236/nm.2011.22011

43. Matsui T, Fujimoto T, Endo Y, et al. Neck neuromuscular treatment for depressive disorders with cervical muscular tension or chronic neck pain-a pilot study. Open J Psychiatr. 2012;2(01):61-67. doi:10.4236/ojpsych.2012.21009

44. Kaka B, Ogwumike OO, Adeniyi FA. Efficacy of neck stabilisation and dynamic exercises on pain intensity, depression and anxiety among patients with non-specific neck pain. Physiotherapy. 2016;102:e2. doi:10.1016/j.physio.2016.10.005

45. Nazari G, Bobos P, Billis E, MacDermid JC. Cervical flexor muscle training reduces pain, anxiety, and depression levels in patients with chronic neck pain by a clinically important amount: a prospective cohort study. Physiother Res Int. 2018;23(3):e1712. doi:10.1002/pri.1712

46. Laborit H. On the mechanism of activation of the hypothalamo-pituitaryadrenal reaction to changes in the environment (the 'alarm reaction'). Resuscitation. 1976;5(1):19-30. doi:10.1016/0300-9572(76)90017-4
47. Steimer T. The biology of fear- and anxiety-related behaviors. Dialogues Clin Neurosci. 2002;4(3):231-249.

48. Chebyshev N, Berechikidze I, Philippova A, Lazareva Y, Gorozhanina E. The significance of the system approach in the study of the general adaptation syndrome' reactions. Georgian Med News. 2019;(292-293):102-108.

49. Tomasi J, Zai CC, Zai G, Kennedy JL, Tiwari AK. Genetics of human startle reactivity: a systematic review to acquire targets for an anxiety endophenotype. World J Biol Psychiatry. 2020;1-29. doi:10.1080/15622975.2020.1834619

50. Rutherford AV, Tanovic E, Bradford DE, Joormann J. Psychophysiological correlates of anxious apprehension: trait worry is associated with startle response to threat. Int J Psychophysiol. 2020;158:136-142. doi:10.1016/j.ijpsycho.2020.09.020

51. Schlund MW, Cataldo MF. Amygdala involvement in human avoidance, escape and approach behavior. Neuroimage. 2010;53(2):769776. doi:10.1016/j.neuroimage.2010.06.058

52. LeDoux JE. The Emotional Brain: The Mysterious Underpinnings of Emotional Life. New York: Simon \& Schuster; 1996.

53. LeDoux JE. Anxious: Using the Brain to Understand and Treat Fear and Anxiety. New York, NY: Viking; 2015.

54. Shahidi B, Haight A, Maluf K. Differential effects of mental concentration and acute psychosocial stress on cervical muscle activity and posture. J Electromyogr Kinesiol. 2013;23(5):1082-1089. doi:10.1016/j.jelekin.2013.05.009
Psychology Research and Behavior Management

\section{Publish your work in this journal}

Psychology Research and Behavior Management is an international, peer-reviewed, open access journal focusing on the science of psychology and its application in behavior management to develop improved outcomes in the clinical, educational, sports and business arenas. Specific topics covered in the journal include: Neuroscience, memory and decision making; Behavior modification and management; Clinical

\section{Dovepress}

applications; Business and sports performance management; Social and developmental studies; Animal studies. The manuscript management system is completely online and includes a very quick and fair peer-review system, which is all easy to use. Visit http://www. dovepress.com/testimonials.php to read real quotes from published authors. 